\title{
Jet-induced star formation by a microquasar
}

\author{
I. F. Mirabel ${ }^{1,2}$, S. Chaty ${ }^{1,3}$, L.F. Rodríguez ${ }^{4}$ and M. Sauvage ${ }^{1}$ \\ ${ }^{1}$ Laboratoire AIM, CEA/DSM/Irfu/Service d'Astrophysique, Centre de Saclay, Bât. 709, \\ FR-91191 Gif-sur-Yvette Cedex, France \\ email: felix.mirabel@cea.fr \\ ${ }^{2}$ Instituto de Astronomía y Física del Espacio C.C. 67, Suc. 28. 1428, Buenos Aires, Argentina \\ ${ }^{3}$ Institut Universitaire de France, 103, boulevard Saint-Michel, 75005 Paris, France \\ ${ }^{4}$ Centro de Radioastronomía y Astrofísica, UNAM, Campus Morelia, Morelia, Michoacán \\ 58190, México
}

\begin{abstract}
Theoretical and observational work show that jets from AGN can trigger star formation. However, in the Milky Way the first -and so far- only clear case of relativistic jets inducing star formation has been found in the surroundings of the microquasar GRS 1915+105. Here we summarize the multiwavelength observations of two compact star formation IRAS sources axisymmetrically located and aligned with the position angle of the sub-arcsec relativistic jets from the stellar black hole binary GRS 1915+105 (Mirabel \& Rodríguez 1994). The observations of these two star forming regions at centimeter (Rodríguez \& Mirabel 1998), millimeter and infrared (Chaty et al. 2001) wavelengths had suggested -despite the large uncertainties in the distances a decade ago- that the jets from GRS 1915+105 are triggering along the radio jet axis the formation of massive stars in a radio lobe of bow shock structure. Recently, Reid et al. (2014) found that the jet source and the IRAS sources are at the same distance, enhancing the evidence for the physical association between the jets from GRS 1915+105 and star formation in the IRAS sources. We conclude that as jets from AGN, jets from microquasars can trigger the formation of massive stars, but at distances of a few tens of parsecs. Although star formation induced by microquasar jets may not be statistically significant in the Milky Way, jets from stellar black holes may have been important to trigger star formation during the re-ionization epoch of the universe (Mirabel et al. 2011). Because of the relative proximity of GRS 1915+105 and the associated star forming regions, they may serve as a nearby laboratory to gain insight into the physics of jet-trigger star formation elsewhere in the universe.
\end{abstract}

Keywords. jets, microquasars, stars: formation

\section{Observations}

The microquasar GRS 1915+105 frequently produces relativistic jets with large kinetic energy, reaching $10^{43} \mathrm{ergs}$ (Rodríguez \& Mirabel 1999). To understand where in the interstellar medium is this energy being dissipated, Rodríguez \& Mirabel (1998) carried out radio studies of the surroundings of GRS 1915+105. They found the intriguing possibility of a physical connection of GRS 1915+105 with two infrared/radio sources that appear symmetrically located with respect to GRS $1915+105$ and aligned with the position angle of the relativistic jets.

In Figure 1 are shown a mosaic of the surroundings of GRS $1915+105$ and the maps of the two compact infrared/radio sources IRAS $19124+1106$ and IRAS $19132+1035$ at $\lambda 20 \mathrm{~cm}$ obtained with the VLA. In IRAS $19132+1035$ there is to its northwest edge a remarkable feature with a non-thermal spectrum of index -0.8. This feature points back to GRS 1915+105, and as proposed by Rodríguez \& Mirabel (1998) and Chaty et al. (2001), this non-thermal feature could be produced by the interaction of the jets from 


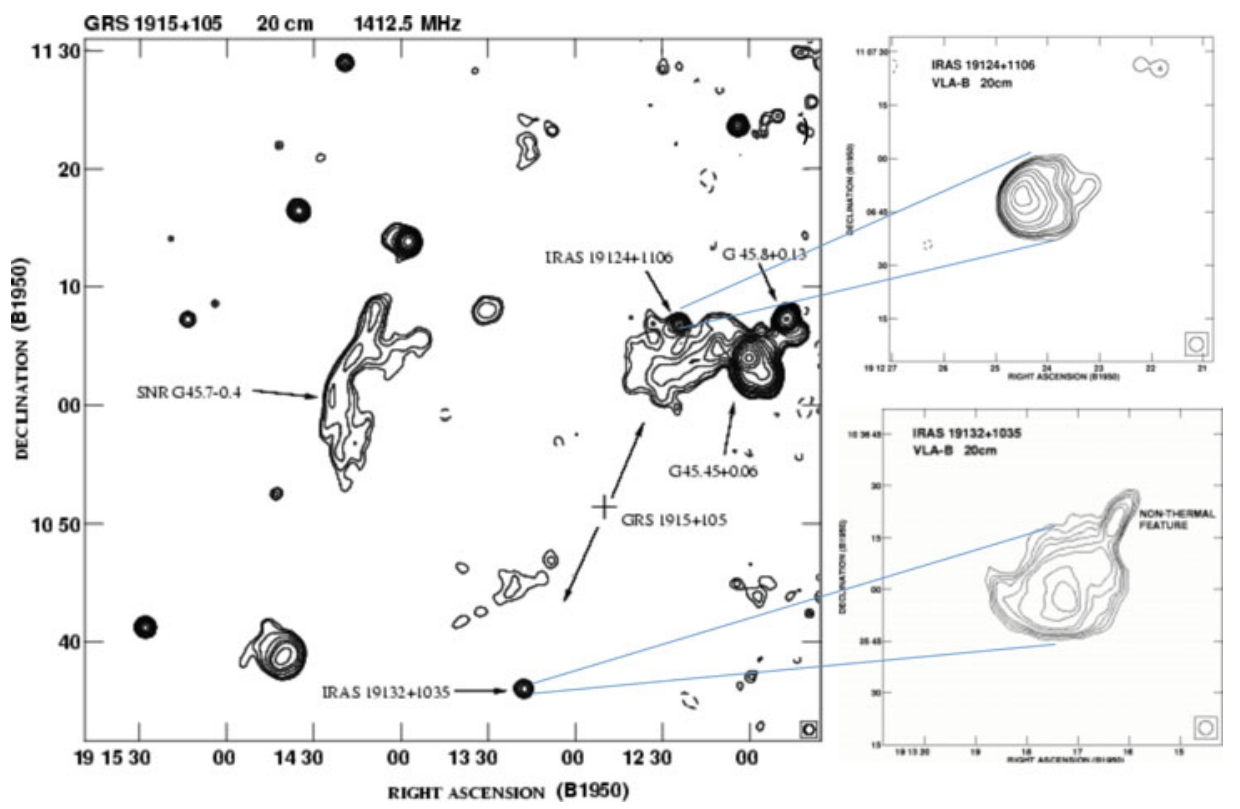

Figure 1. Left: Map of the surroundings of GRS $1915+105$, taken with the VLA-C at $20 \mathrm{~cm}$. The arrows emerging from GRS 1915+105 indicate the position angle of the sub-arcsec relativistic ejecta. Along the axis of the jets from GRS 1915+105 are found two compact infrared/radio sources, IRAS $19124+1106$ and IRAS $19132+1035$, located at 40-50 parsecs from the microquasar jet source. Contour levels are -3, 3, 4, 6, 10, 15, 20, 30, 40,60, 100, 200, 400 and 800 $\mathrm{mJy} /$ beam. The half power contour of the beam is shown in the bottom right corner. Right: VLA maps at $20 \mathrm{~cm}$ of the star formation IRAS sources. Note the bow shock structure and non-thermal feature in IRAS $19132+1035$ that points to GRS $1915+105$. The half power contour of the beam, with diameter of 4 ", is shown in the bottom right corner. Contours are $-4,4$, $6,8,10,15,20,40,60,100,200,300$, and 400 times $0.05 \mathrm{mJy} /$ beam.

GRS 1915+105 and the HII region/compact molecular cloud associated with the IRAS source.

Chaty et al. (2001) carried out observations at millimeter (IRAM $30 \mathrm{~m}$ ), and infrared (ISO, UKIRT, ESO/MPI $2.2 \mathrm{~m}$ ) wavelengths in both line and continuum emission. Using millimeter tracers of $\mathrm{CO}, \mathrm{CS}$ and $\mathrm{HCO}^{+}$strong density enhancements are found towards the non-thermal radio feature in the NW of IRAS $19132+1035$. At infrared wavelengths strong hydrogen recombination lines and weak lines of molecular hydrogen are observed in that IRAS source. In Figure 2 is shown the ISO map at 7 microns, and in Figure 3 the Herschel map at 160 microns of IRAS 19132+1035. Both show in the thermal dust emission a similar bow shock structure as seen in the thermal emission at radio waves.

\section{Evidences for jet induced star formation in GRS $1915+105$}

The strongest lines of evidence supporting a physical interaction between the jets from GRS 1915+105 and the compact star forming regions are: (1) the axisymmetric locations of the two compact star forming sources at nearly the same position angle as the sub-arcsec ejections observed at radio wavelengths, (2) the spatial coincidence of the non-thermal radio feature with the inner edge of the southern source IRAS $19132+1035$, as well as the orientation of this small non-thermal feature along the axis of the GRS $1915+105$ jets, (3) the location of the highest gas densities in IRAS 19124+1106 and IRAS $19132+1035$ on the sides closest to GRS $1915+105$ and, in the case of IRAS 


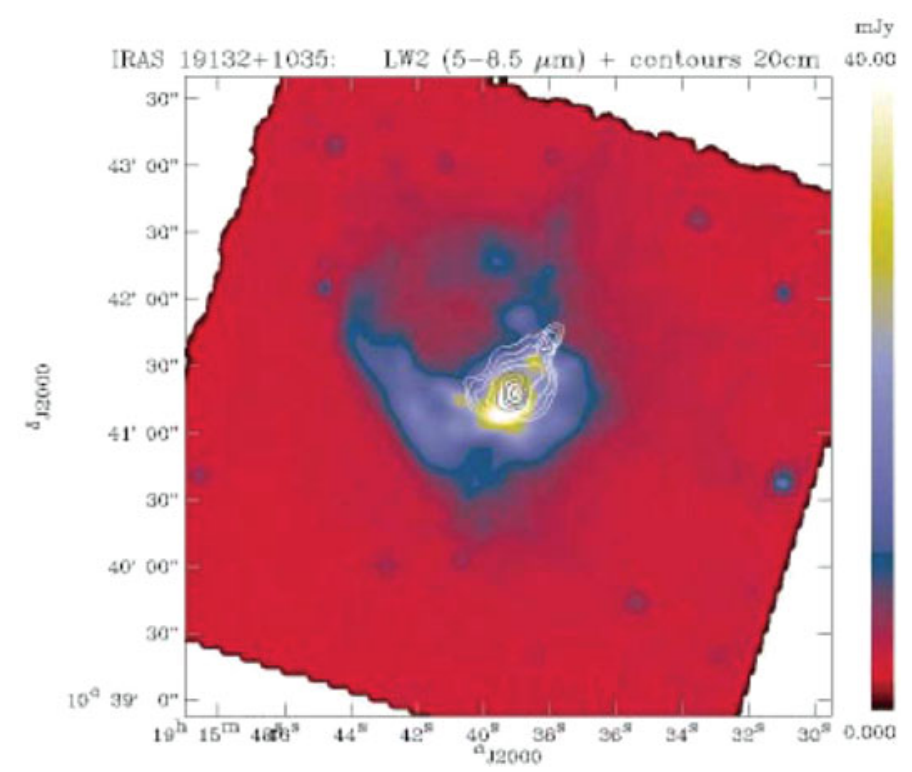

Figure 2. ISO map of the source IRAS $19132+1035$, at $\lambda=7 \mu m$, taken with the LW2 filter. Superimposed are the $20 \mathrm{~cm}$ radio contours at the levels $0.2,0.4,0.7,1.2,1.8,2.5,3,4$ and 5 mJy. Note the bow shock structure in both, the radio and $\lambda=7 \mu \mathrm{m}$ maps.

$19132+1035$, close to the non-thermal jet, (4) the bow shock structure in the portion of IRAS 19132+1035 most distant from GRS 1915+105, and (5) the compactness of the molecular clouds associated with the IRAS sources.

\section{Discussion}

At the new distance determination of the jet source and IRAS sources of $8.6(+2.0 /-$ 1.6) kpc, and jet inclination with the line of sight of $60 \pm 5$ degrees (Reid et al. 2014), the IRAS sources are at 40-50 pc from GRS 1915+105. Since all these objects are within 1 degree from the Galactic plane, one may ask why there is no triggered star formation closer to GRS 1915+105. Two properties of GRS 1915+105 can account for this: (1) the precession of the jets is less than 10 degrees (Rodríguez \& Mirabel 1999), small enough to remain inside the lobes (Chaty et al. 2001; Kaiser 2006); (2) The accretion to the 12.4 solar mass black hole is by Roche lobe overflow from a red giant star, which may have lasted tens, even hundreds of thousand years, the time for the donor star to cross the Hertzsprung gap and remain in the giant branch. Therefore as proposed by Chaty et al. (2001), a large cavity in the form of a tunnel could have been produced, which would explain the ballistic motions of the repeated ejections from GRS 1915+105 observed by Rodríguez \& Mirabel (1999).

The high energy of the ejecta, low angular precession of the jet axis, and the possibility that GRS $1915+105$ has been an active high energy jet source for tens of thousands years, suggest that the jets from GRS 1915+105 could have overpressured the interstellar gas collecting it and enhancing its density to form a molecular cloud that would ultimately collapse forming massive stars. The compactness and displacement of the high density molecular gas associated to the IRAS sources as observed by Chaty et al. (2001) using several molecular transitions, supports the idea that the jets not only may induce by overpressure the formation of stars, but even induce the formation of the progenitor 


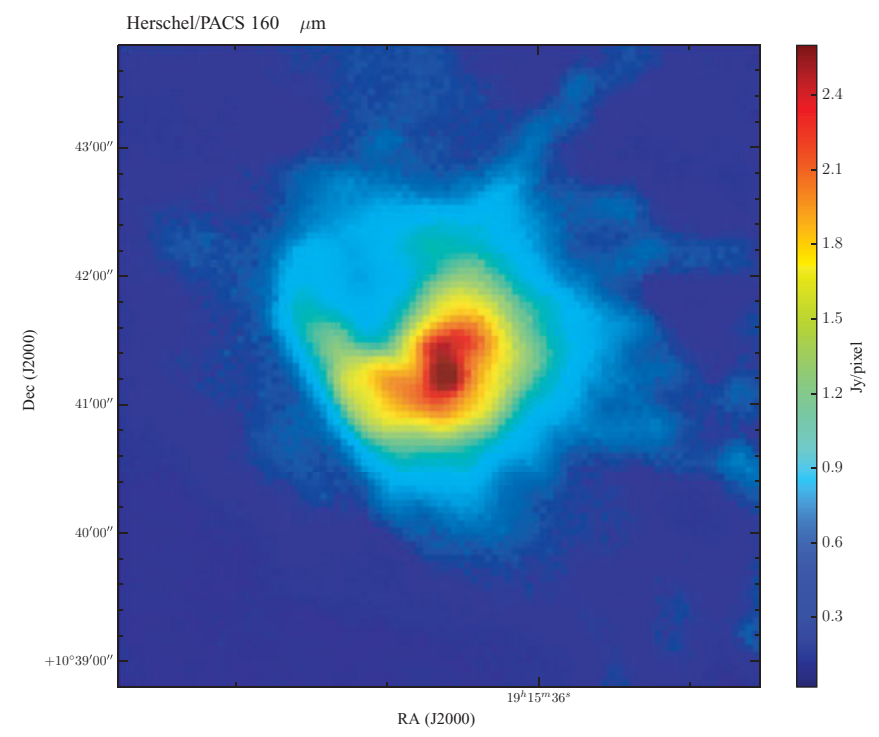

Figure 3. Herschel map of IRAS $19132+1035$ at $\lambda=160 \mu \mathrm{m}$. The data obtained under the Galactic Plane key project (P.I. S. Molinari) were reduced by one of us. Note the filamentary feature pointing to the NW as the non-thermal feature in the $20 \mathrm{~cm}$ maps of Figures 1 and 2.

molecular cloud. If this is the case, the lobes of GRS $1915+105$ and the stars formed in them should be moving away from GRS 1915+105. Our preliminary follow up observations at radio waves suggest in the ionized gas of IRAS $19132+1035$ the possibility at 3-4 sigma of proper motions away from GRS 1915+105.

It has been proposed that jet-triggered positive feedback from supermassive black holes produce global star formation, generating and enhancing ultraluminous starbursts with top heavy IMF (Silk 2005). On the other hand, Mirabel et al. (2011) have proposed that due to cosmic chemical evolution, during the re-ionization epoch of the universe the numbers and energy feedback from stellar black hole high-mass X-ray binaries should be greatly enhanced relative to their numbers and energy feedback in the present epoch. Given the much larger densities of the IGM at redshifts greater than $z=10$, in addition to jets from putative supermassive black holes, jet induced star formation by stellar black holes should have been important to induce star formation. In this context, it will be interesting to probe whether the star formation in IRAS $19132+1035$ is top heavy, as predicted by theoretical models.

\section{References}

Chaty, S., Rodríguez, L. F., Mirabel, I. F., Geballe, T., \& Fuchs, Y. 2001, A\&A, 366, 1041

Kaiser, C. R. 2006, MNRAS, 367, 1083

Mirabel, I. F., Dijkstra, M., Laurent, P., Loeb, A., \& Pritchard, J. R. 2011, A\&A, 528, A149

Mirabel, I. F. \& Rodríguez, L. F. 1994, Nature, 371, 46

Reid, M. J., McClintock, J. E., Steiner, J. F., et al. 2014, ApJ, 796, 2

Rodríguez, L. F. \& Mirabel, I. F. 1998, A\&A, 340, L47 (RM98)

Rodríguez, L. F. \& Mirabel, I. F. 1999, ApJ, 511, 398

Silk, J. 2005, MNRAS, 364, 1337 